Japanese Psychological Research

1967, Vol. 9, No. 2, 78-84

\title{
INTRADIMENSIONAL AND EXTRADIMENSIONAL SHIFTS AS A FUNCTION OF STIMULUS CHANGE AND OVERTRAINING ${ }^{1}$
}

\author{
TAKESHI SUGIMURA
}

Nara University of Education

\begin{abstract}
To assess the effect of stimulus similarity between original and shift tasks on intradimensional (IN) and extradimensional (EX) shifts under 2 levels of training, 4 to 6 years old children were trained on a 2 -dimensional discrimination task. The stimulus change was determined by whether the stimulus values in relevant and irrelevant dimensions of an original task were the same as those of a shift task or not: Pattern 1 (same stimuli in relevant and irrelevant dimensions, same-same), Pattern 2 (same in relevant but different in irrelevant, same-different), Pattern 3 (different-same), and Pattern 4 (different-different). The results were that (a) the IN shift was easier than the EX shift under all pattems, (b) the EX shift was faster under Patterns 2 and 4 than under Patterns 1 and 3, and (c) the IN shift was faster under Pattern 4 than under Pattern 2. The results were discussed with reference to 2-stage theories which assume the transfer effect of mediating or attentional responses and original instrumental responses.
\end{abstract}

The purpose of the present experiment was to assess the effect of stimulus similarity between original and shift discrimination tasks on intradimensional (IN) and extradimensional (EX) shifts under two different levels of original learning.

A number of experiments have been recently reported with respect to the INEX comparison with or without overtraining of original discrimination task, and they have given rise to important developments in the theory of discirmination learning (House \& Zeaman, 1962; Kendler \& Kendler, 1962; Mackintosh, 1965). A critical difference between IN and EX shifts is that in IN shift $S$ is required to respond to the same dimension that was relevant during original task, while in EX shift the previously relevant dimension is no longer relevant and a previously irrelevant dimension becomes relevant. However,

1 The author is indebted to Dr. S. Iwahara for his valuable comments to an early draft of the present paper, and Miss Y. Fujimoto for her assistance in collection and analysis of the present data. more detailed examination of previous experiments reveals that various patterns in IN and EX shifts are employed for the comparison of shift behavior and that the effect of stimulus change between original and shift tasks is not considered in comparing IN with EX shifts. Some studies (e.g., Kendler \& Kendler, 1959) use the same stimuli in the shift as in the original task, and others (e.g., Eimas, 1966; Shepp \& Eimas, 1964) use the different stimuli from the relevant and irrelevant dimensions in the original task. So far, no precise comparison of the ease of various patterns in IN and EX shifts seems to be performed when the similarity between original and shift stimuli is systematically varied. The primary purpose of this experiment, therefore, was to offer some empirical evidences concerning with the effect of stimulus change on IN and EX shifts.

Recently, mediational or two-stage theories of discrimination learning have been developed based mainly on the findings with respect to the overtraining reversal effect and the IN-EX comparison. 
All two-stage theories coincide with the assumption that some dimension-specific mediator intervenes between external stimulation and overt instrumental responses, although the nature of the mediator is somewhat different from theorist to theorist, for example, observing responses (House \& Zeaman, 1962), implicit verbal responses (Kendler \& Kendler, 1962), or selective attention (Mackintosh, 1965) to the relevant stimulus dimension. These mechanisms may be divided into verbal and nonverbal mediations (Eimas, 1966), or into additive and subtractive (Tighe \& Tighe, 1966). According to the twostage theory, the first stage of discrimination learning involves the establishment of mediating responses to the relevant dimension, and the second stage involves the acquisition of overt instrumental responses to the stimulus values of the relevant dimension. A further important assumption of the theory is that mediating responses transfer from one discrimination task to another, just as overt instrumental responses. It would be expected from this assumption that positive transfer of mediating responses occurs when the same stimulus dimension is relevant both in original and shift tasks, while negative transfer occurs when the relevant dimension in original task becomes irrelevant in the shift task. Shepp and Eimas (1964) showed that rats learned IN shift faster than EX shift using the shift task consisted of different stimuli from the relevant and irrelevant stimulus dimensions in the original task, indicating that mediating (observing) responses acquired in original discrimination transfered to the learning of shift discrimination task.

It is suggested from the two-stage theory that ease of shift discrimination learning depends upon the amount of transfer of mediating responses and original instrumental responses to the shift task, that is, upon the relative strength of mediating responses to the relevant stimulus dimension versus instrumental responses to the stimulus values of the relevant dimension. Recently, Trabasso, et al. (1966) succeeded in assessing the separate effects of attentional or mediating responses and instrumental responses on shift discrimination learning by comparing two pairs of shifting: reversal versus intradimensional shifts, and intradimensional versus extradimensional shifts, respectively. Trabasso, et al. inferred the strength of original instrumental responses from the performance difference of the former pair, and the role of attention from that of the latter.

The present experiment aimed directly at comparing various patterns of IN and EX shifts also permits to assess more systematically the relative role of mediating responses and instrumental responses in shift discrimination learning.

\section{METHOD}

Subjects. The $S \mathrm{~s}$ used in the present study were 256 children (134 boys and 122 girls) from four kindergartens and two nursery schools in Nara and Nishiwaki. They were between 48 and 72 months of age with a mean of 61.7 months. They were assigned to one of the 16 treatment groups in order of entering into the experimental room. As the mean ages of the 16 groups ranged from 59.1 months to 64.3 months and no large difference in sex distribution was found among groups, all groups were regarded as homogeneous with respect to age and sex.

Stimulus materials. A pack of $4018 \times 12.5 \mathrm{~cm}$ stimulus cards was used for the learning of original discrimination and four packs of such cards were for the learning of shift discrimination. The stimuli varied on two (form and color) dimensions with two values within each dimension, were mounted on each card, side by side. As was shown in Fig. 1, forms in the original task were square and circle, and those in the shift task were square, circle, equilateral triangle, and star. Colors were red and yellow in the original task, red, yellow, blue, and gray in the shift task. The areas of forms were so constructed that they seem to be equal 
in appearance; the circle's diameter was $6.0 \mathrm{~cm}$, the square's side $5.5 \mathrm{~cm}$, the equilateral triangle's height $5.7 \mathrm{~cm}$, and the circumscribed circle's diameter of the star $7.0 \mathrm{~cm}$. Each pack of 40 stimulus cards consisted of four types of stimulus pair with 10 each. Two types of the four were those as shown in Fig. 1 and the remaining two were those with the reverse left-right position of the arrangements in Fig. 1. The stimulus cards were presented one at a time in random order with restriction that the same type of pair was not given in succession.

Fig. 1 presents an illustration of the stimuli and one of the reinforcement patterns. There were four patterns of the stimulus change in IN and EX shifts, respectively: IN-1, IN-2, IN-3, and IN-4 in the IN shift, and EX-1, EX-2, EX-3, and EX-4 in the EX shift. The stimulus change in the present experiment was determined in terms of whether the stimulus values in the relevant and irrelevant dimensions of original discrimination task were the same as those of the shift discrimination task or not; In the IN-1 and EX-1 the same stimulus values both in the relevant and irrelevant dimensions were used in original task as well as in shift task, in the IN-2 and EX-2 the stimulus values of the relevant dimension in original task were retained in shift task but those of the irrelevant dimension were changed, in the IN-3 and EX-3 the stimulus values of the relevant dimension in original task were changed but those of the irrelevant dimension were retained, and in the IN-4 and EX-4 the different stimulus values from the relevant and irrelevant dimensions of original task were used in shift task.

Procedures. A $4 \times 2 \times 2$ factorial design was used with four patterns of the stimulus change, two different amounts of original training, and two types of shifting. The $S$ s were tested individually. After $S$ was seated, a singledimensional discrimination task consisting of two digits ( 1 and 2) was given in order to eliminate $S$ 's position habit and to see whether he understood the proper way to respond. Then, $E$ placed the stimulus cards before the $S$ and read the following instructions:

Now, I'm going to show you many cards one at a time and play a card game with you. Each of the cards has two figures.

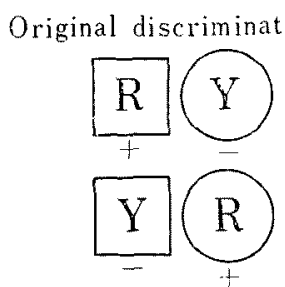

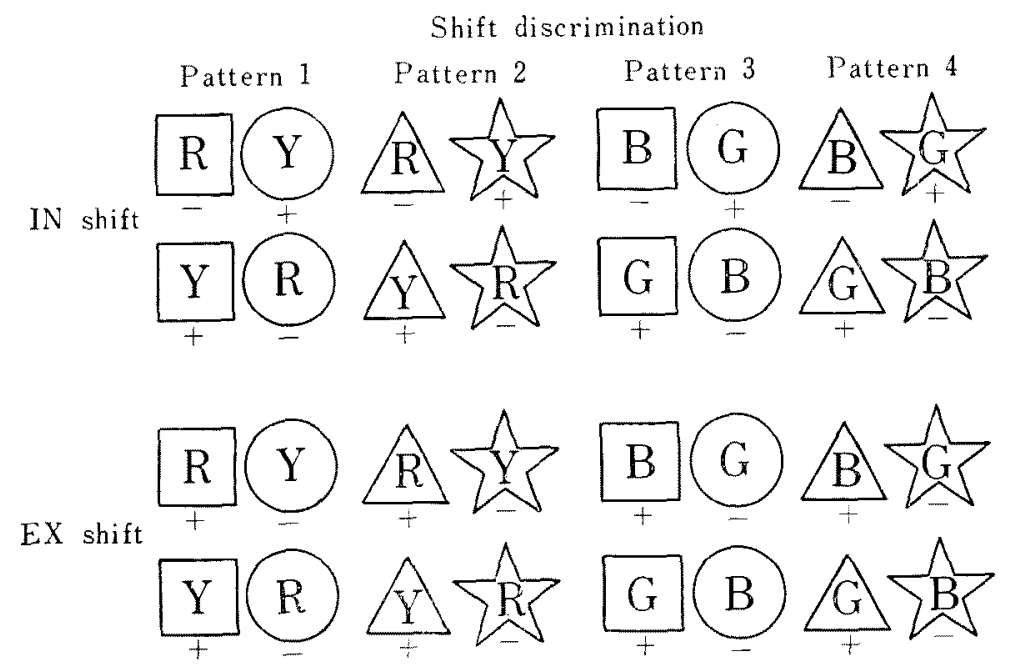

Fig. 1. An illustration of the stimuli and one of the reinforcement patterns. 
One of them is always right and the other is always wrong. Your task is to decide which one be right each time and to point at it. If you choose the right figure, I will say "Right" or "Hit" after each trial. But if you choose the wrong one, I will say "Wrong" or "Miss". I want you try to get as many "Rights" or "Hits" as possible.

After reading the instructions, the original task was given either to a criterion of nine correct responses out of 10 successive trials or to additional 30 overtraining trials after the criterion. Each of the four stimulus values (red, yellow, circle, and square) was equally assigned as a positive value. If $S$ failed to meet the criterion of original discrimination within 40 trials, a special training procedure was instituted. $E$ pointed at the positive stimulus and said, "This is the correct one," but did not inform the name of color or form of positive stimulus values. This special training was judged necessary due to a relatively high percentage (about 20\%) of $S \mathrm{~s}$ who failed to reach the criterion within 50 trials in a pilot study. Additional $18 \mathrm{~S}$ s were dropped from the experiment for failure to learn the original discrimination task within 60 trials in spite of the special training.

Immediately after the original discrimination was completed, the $S$ s were given a shift discrimination task without any instructions about the nature of the shift task until a criterion of nine out of 10 correct responses was attained for all $S$ s. As illustrated in Fig. 1, the four patterns of stimulus change were employed in each of the IN and EX shifts. The relevant dimensions and the positive stimulus values in the dimension were counterbalanced within each group, for example, out of the $16 \mathrm{IN}-1$ $S_{\mathrm{S}}$ who were trained to the criterion, four $S_{\mathrm{s}}$ were trained on the red stimulus and were shifted to the yellow stimulus, four $S$ s on the yellow to the red, four $S \mathrm{~s}$ on the circle to the square, and the remaining four $S \mathrm{~s}$ on the square to the circle.

Throughout the experiment, the stimulus cards were presented manually one at a time depending upon the $S$ 's response rate, and a noncorrection procedure was used. If $S$ did not attain the criterion within the presentation of 40 stimulus cards, the same cards were given once more. The shift task was given to the criterion or to 80 trials.

\section{Results}

Original discrimination. Table 1 presents mean errors ( $\sqrt{\mathrm{X}+0.5}$ transformation) to criterion in the original task. To test whether the groups differed on the original task, a $4 \times 2 \times 2$ analysis of variance was performed, indicating that neither of the main effects nor the interactions were significant, and therefore, it may be assumed that the groups were approximately equated with each other.

Shift discrimination. Mean errors in the shift discrimination are given in Table 2. A $4 \times 2 \times 2$ analysis of variance was performed to evaluate the effects of the experimental variables on learning the shift task. The results are shown in Table 3 which indicated that (a) the IN shift was easier than the EX shift, (b) the effect of stimulus change was statistically significant, and (c) there was a significant interaction between type of shift and pattern of stimulus change. The significant pattern effect means that the shift discrimination under Pattern 4 was significantly easier than under Pattern $1(p<$ $.01)$ and Pattern $3(p<.05)$. The shift by stimulus change $(\mathrm{T} \times \mathrm{P})$ interaction means that difference in errors between IN and EX shift discriminations was not equal under the four varying stimulus change patterns or pattern differences in error scores were not identical under IN and EX shift discriminations. A series of $t$ tests yielded that $p<.01$ for the comparison of Patterns 3 and 4 , and $p<.001$ for the comparisons of Patterns 1 and 2, Patterns 1 and 4, and Patterns 2 and 3, respectively, in the EX shift, while in the IN shift only the difference between Patterns 2 and 4 was significant $(p<.05)$. Although the IN shift was superior to the EX shift under all 
TABLE 1

Mean errors to criterion in original discrimination by the use of $\sqrt{\mathrm{X}+0.5}$ transformed scores

\begin{tabular}{|c|c|c|c|c|c|c|}
\hline & & \multicolumn{5}{|c|}{$\begin{array}{l}\text { Pattern of stimulus change } \\
\text { in shift discrimination }\end{array}$} \\
\hline \multirow{3}{*}{\multicolumn{2}{|c|}{$\begin{array}{l}\text { Relevant dimension } \\
\text { in original task } \\
\text { Irrelevant dimension } \\
\text { in original task }\end{array}$}} & 1 & 2 & 3 & 4 & \multirow{3}{*}{ Mean } \\
\hline & & Same & Same & Diff. & Diff. & \\
\hline & & Same & Diff. & Same & Diff. & \\
\hline \multirow{3}{*}{ IN shift } & C & 2.21 & 1.95 & 2.42 & 1.90 & 2.12 \\
\hline & OT & 1.94 & 2.22 & 2.16 & 2.63 & 2.24 \\
\hline & Mean & 2.07 & 2.08 & 2.29 & 2.26 & 2.18 \\
\hline \multirow{4}{*}{ EX shift } & C & 2.23 & 2.27 & 2.24 & 2.13 & 2.22 \\
\hline & OT & 2.51 & 2.31 & 2.01 & 1.84 & 2.17 \\
\hline & Mean & 2.37 & 2.29 & 2.13 & 1.98 & 2.19 \\
\hline & Mean & 2.22 & 2.18 & 2.21 & 2.12 & \\
\hline
\end{tabular}

TABLE 2

Mean errors in shift discrimination by the use of $\sqrt{\mathrm{X}+0.5}$ transformed scores

\begin{tabular}{|c|c|c|c|c|c|c|}
\hline \multirow{4}{*}{\multicolumn{2}{|c|}{$\begin{array}{l}\text { Relevant dimension } \\
\text { in original task } \\
\text { Irrelevant dimension } \\
\text { in original task }\end{array}$}} & \multicolumn{5}{|c|}{$\begin{array}{l}\text { Pattern of stimulus change } \\
\text { in shift discrimination }\end{array}$} \\
\hline & & 1 & 2 & 3 & 4 & \multirow{3}{*}{ Mean } \\
\hline & & Same & Same & Diff. & Diff. & \\
\hline & & Same & Diff. & Same & Diff. & \\
\hline \multirow{3}{*}{ IN shift } & $\mathrm{C}$ & 1.78 & 2.41 & 1.43 & 1.37 & 1.75 \\
\hline & OT & 1.41 & 1.97 & 1.32 & 1.32 & 1.50 \\
\hline & Mean & 1.59 & 2.19 & 1.37 & 1.34 & 1.63 \\
\hline \multirow{4}{*}{ EX shift } & C & 4.48 & 3.54 & 4.03 & 3.57 & 3.90 \\
\hline & OT & 4.77 & 2.59 & 4.70 & 2.80 & 3.71 \\
\hline & Mean & 4.62 & 3.06 & 4.36 & 3.18 & 3.81 \\
\hline & Mean & 3.11 & 2.62 & 2.87 & 2.26 & \\
\hline
\end{tabular}

TABLe 3

Analysis of variance of mean errors in shift discrimination

\begin{tabular}{lrrc}
\hline \multicolumn{1}{c}{ Source } & $d f$ & $M S$ & $F$ \\
\hline Type of shift $(\mathrm{T})$ & 1 & 304.68 & $105.06 * * *$ \\
Pattern of stimulus & & & \\
$\quad$ change (P) & 3 & 8.31 & $2.87^{*}$ \\
Overtraining (O) & 1 & 3.03 & 1.04 \\
$\mathrm{~T} \times \mathbf{P}$ & 3 & 17.09 & $5.89^{* * *}$ \\
$\mathrm{~T} \times \mathrm{O}$ & 1 & .05 & - \\
$\mathrm{P} \times \mathrm{O}$ & 3 & 2.92 & 1.00 \\
$\mathrm{~T} \times \mathbf{P} \times \mathrm{O}$ & 3 & 2.43 & - \\
Error (Subjects) & 240 & 2.90 & \\
Total & 255 & & \\
$\quad * p<.05 \quad * * * p<.001$ & &
\end{tabular}

patterns, an inspection of Table 2 revealed that the IN-EX difference was larger under Patterns 1 and 3 than under Pattern 2. As the main effect of overtraining and the interactions associated with overtraining were insignificant, neither facilitative nor inhibitory effects of overtraining were confirmed. As far as sample means were concerned, error responses became less with overtraining under all patterns in the IN shift, but in the EX shift errors became less under Patterns 2 and 4 and greater under Patterns 1 and 3. 


\section{Discussion}

The findings that $S$ s learn more rapidly the IN shift than the EX shift are in line with a mediational view; mediating responses to the dimension developed during the original discrimination remain relevant in the IN shift, while new mediating responses must be acquired in the EX shift. However, the Kendler's (1962) position that assumed the relation between the ability to utilize mediating responses and developmental or age level seems not to fit the present results. According to the Kendlers' theory, there is a transitional period from " nonmediator" to " mediator" in the course of development, and the period is considered to be ranged in age from four to six years. In fact, previous studies using children within the period reported that no difference was found between reversal and nonreversal shifts (Kendler \& Kendler, 1959) and that 50 percent of the $S_{\mathrm{s}}$ responded in a reversal manner during the optional shift task (Kendler, Kendler, \& Learnard, 1962). Contrary to these results, four to six years old children in the present study learned the IN shift faster than the EX shift. In explaining the present findings, therefore, it scems to be better to assume an attentional or perceptual mediation (House \& Zeaman, 1962; Mackintosh, 1965) which is considered to be unrelated to the developmental or age level. Further research is needed about the topics on perceptual versus verbal mediation (e.g., McConnell, 1964).

Although ease of the IN and EX shifts is largely dependent upon the stimulus change (pattern) as shown in Table 2, only one or two patterns in each shift have been employed for comparing IN with EX shifts in previous studies. For example, House and Zeaman (1962) used three patterns like as the IN-1, IN-3, and EX-3 in the present experiment, Youniss and Furth (1965) used similar shifts to the IN-2 and EX-3, and Eimas (1966) used new stimuli from the relevant and irrelevant dimensions, i.e., the IN-4 and EX-4 in the present study. It is evident from the present findings that the stimulus similarity between original and shift tasks must be considered in the comparison of IN with EX shifts.

Recently, Uhl (1966) reported using college students that (a) the IN shift using identical stimuli to those used in training (similar to the $\mathrm{IN}-\mathrm{l}$ in the present study) took fewer trials to reach criterion than the IN shift using different stimuli (the IN-4), and (b) the EX shift using identical stimuli on the previously relevant dimension (the $\mathrm{EX}-1$ ) took more trials to reach criterion than the EX shift using different stimuli (the EX-4). The latter results are consistent with the present findings, while the former are in conflict with the present. Uhl (1966) explained the result (a) by hypothesizing that the mediating responses developed during training might be weakened by generalization decrement when different stimuli were used in the shift task. However, the present difference in the opposite direction cannot be attributed to the generalization concept.

Harrow (1964) found the superiority of the IN-3 (within-dimensional shift in his term) to the IN-1 (response reversal), and explained the results in terms of a 'paying-selective-attention' theory; that is, $S$ s learn to pay selective attention to a general dimension of the stimuli during original task, and they continue to pay selective attention to that aspect of the stimuli, even when the specific stimulus values formerly present are no longer there. On the other hand, Isaacs and Duncan (1962) reported that the IN-3 (nonreversal same in their term) was learned faster than the IN-2 (reversal) did. The results were accounted for by a extinction theory in that the IN-shift was retarded because the previously learned responses must be extingushed in the IN-2 shift. Both the 'paying-selective-attention' and extinc- 
tion theories seem to fit to the present results in the IN shift. When these theories are compared with the two-stage theories of discrimination learning, the selective attention and the extinction theory approximately corresponded with mediating responses and overt instrumental responses in the two-stage model, respectively.

According to the two-stage model, ease of discrimination shifts is a function of the relative strength of mediating responses and instrumental responses. The model assumes that positive transfer of mediating responses would occur in the IN shift, while negative transfer in the EX shift. However, transfer effects of original instrumental responses are dependent upon the pattern of stimulus change. Negative transfer would be expected in the IN-1 and IN-2 shifts because the same relevant stimuli were used both in the original and shift tasks, while there is no transfer effect of the instrumental responses in the IN-3 and IN-4 shifts because different stimuli in the relevant dimension were used in both discrimination tasks. On the other hand, negative transfer would be expected in the EX-1 and EX-3 shifts because the original instrumental responses to the stimuli must be extinguished, while the instrumental responses need not be extinguished in the EX-2 and EX-4 shifts. The present results are consistent with these reasonings. If it is attempted to assess the pure transfer effect of mediating or observing responses on the IN and EX shifts, new stimuli from the previously relevant and irrelevant dimensions, e.g., Pattern 4 in the IN and EX shifts, must be used to eliminate the effect of original instrumental responses (Eimas, 1966; Shepp \& Eimas, 1964).

Although failure to obtain facilitative effects of overtraining in the IN shift is inconsistent with the prediction from House and Zeaman (1962) and Mackintosh (1965) theories, it may be explained in terms of the 'floor' effect operating to prevent further decrease in errors beyond the performance level achieved by the criterion groups.

\section{REFERENCES}

Ermas, P. D. 1966 Effects of overtraining and age on intradimensional and extradimensional shifts in children. 7. exp. child. Psychol., 3, 348-355.

Harrow, M. 1964 Stimulus aspects responsible for the rapid acquisition of reversal shifts in concept formation. 7. exp. Psychol., 67, 330334.

House, B. J., \& Zeaman, D. 1962 Reversal and nonreversal shifts in discrimination learning in retardates. 7. exp. Psychol., 63, 444-451.

IsaAcs, D., \& Duncan, C. P. 1962 Reversal and nonreversal shifts within and between dimensions in concept formation. 7. exp. Psychol, 64, 580-585.

Kendler, H. H., \& Kendler, T. S. 1962 Vertical and horizontal processes in problem solving. Psychol. Rev., 69, 1-16.

Kendler, T.S., \& KendLeR, H. H. 1959 Reversal and nonreversal shifts in kindergarten children. 7. exp. Psychol., 58, 56-60.

Kendler, T. S., Kendler, H. H., \& Learnard, B. 1962 Mediated responses to size and brightness as a function of age. Amer. 7. Psychol., 75, 571-586.

MAckintosh, N. J. 1965 Selective attention in animal discrimination learning. Psychol. Bull., 64, 124-150.

McConnelt, O. L. 1964 Perceptual versus verbal mediation in the concept learning of children. Child Develpm., 35, 1373-1383.

Shepp, S. E., \& Emas, P.D. 1964 Intradimensional and extradimensional shifts in the rat. 7. comp. physiol. Psychol., 57, 357-361.

Trghe, L. S., \& Tighe, T.J. 1966 Discrimination learning: Two views in historical perspective. Psychol. Bull, 66, 353-370.

Trabasso, T., Deutsch, J. A., \& Gelman, R, 1966 Attention in discrimination learning of young children. 7. exp. child Psychol., 4, 9-19.

UHL, N. 1966 Intradimensional and extradimensional shifts as a function of amount of training and similarity between training and shift stimuli. 7. exp. Psychol., 72, 429-433.

Youniss, J., \& Furth, H.G. 1965 Discrimination shifts as a function of degree of training in children. 7. exp. Psychol., 70, 424-427.

(Received May 29, 1967) 